\title{
Lomitapide: a novel drug for homozygous familial hypercholesterolemia
}

Lomitapide (Juxtapid ${ }^{\circledR}$ and Lojuxta ${ }^{\circledR}$; Aegerion Pharmaceuticals, Inc., MA, USA), an orally administered inhibitor of the microsomal triglyceride transfer protein, inhibits the synthesis and secretion of ApoB-containing lipoproteins and, thus, reduces plasma levels of LDL cholesterol (LDL-C). Lomitapide has been approved for the therapy of homozygous familial hypercholesterolemia patients. After a proof-of-concept Phase II trial, lomitapide has been tested in a multinational single-arm, open-label, 78-week, Phase III trial. Lomitapide effectively reduced mean plasma LDL-C levels by $50 \%$ from baseline in 23 adults with homozygous familial hypercholesterolemia over a 26 -week treatment period and this reduction was sustained for an additional 52 weeks of lomitapide treatment. The Phase III trial also demonstrated that $46 \%$ of patients (six out of 13) interrupted or reduced the frequency of apheresis treatments because of an important and stable reduction of LDL-C. Lomitapide was generally well tolerated and the most common adverse events in the Phase III trial were gastrointestinal and hepatic events.

\section{KEYWORDS: HeFH HoFH lipoprotein apheresis lomitapide trials}

Lomitapide (Juxtapid ${ }^{\circledR}$ in the USA and Lojuxta ${ }^{\circledR}$ in Europe), the first fully developed small molecule that inhibits microsomal triglyceride (TG) transfer protein (MTP inhibitor) [1], reduces both LDL and TG plasma levels, and in 2010 received Orphan Drug status for the treatment of hyperlipoproteinemia type IIa in the USA. The drug also received approval from the US FDA in December 2012 and was granted authorization from the European Commission in July 2013 [101,102].

Since the approval of Juxtapid in USA, more than 150 patients with homozygous familial hypercholesterolemia $(\mathrm{HoFH})$ have been treated. Lomitapide represents a novel adjunctive therapy for $\mathrm{HoFH}$.

Familial hypercholesterolemia $(\mathrm{FH})$ is a genetic disease characterized by very high levels of total and LDL cholesterol (LDL-C) and premature cardiovascular disease. Statins are the first-line drugs for the treatment of heterozygous familial hypercholesterolemia ( $\mathrm{HeFH})$. They are able to effectively reduce the LDL-C plasma levels and to reach, even in combination with other lipid-lowering therapies (LLTs), the recommended therapeutic goal owing to their capacity to inhibit cellular cholesterol synthesis, to increase the number of the LDL receptors (LDL-Rs) on the cell membranes and to modify the natural history of the disease.
By contrast, $\mathrm{HoFH}$, a rare disease characterized by extremely high levels of LDL-C, represents a serious, still unsolved clinical problem. To date, available treatments are able to affect only marginally the biochemical phenotype and clinical outcomes of the disease.

The aim of this review is to discuss the evidence-based efficacy, safety, tolerability and clinical use of lomitapide in the context of the treatment of $\mathrm{HoFH}$.

\section{Overview of the available treatments for HoFH}

- HoFH: genetics \& clinics

Autosomal dominant hypercholesterolemia $(\mathrm{ADH})$ is a disorder characterized by elevation of serum cholesterol bound to LDL. ADH is genetically heterogeneous as it can be caused by mutations:

- In $L D L R$, resulting in reduced cell uptake and catabolism of plasma LDL (OMIM 606945) (FH-type 1 [FH-1]) [2];

- In the ApoB-100 (APOB) gene, producing a defective ApoB-100 which binds poorly to the LDL-R (familial defective ApoB, OMIM 603776, FH-type 2) [2];

- In the PCSK9 gene, resulting in abnormal function of the proteolytic enzyme PCSK9
Maria D Panno', Angelo B Cefalù̀' \&. Maurizio R Averna*1 'Dipartimento Biomedico di Medicina Interna e Specialistica, Policlinico 'Paolo Giaccone', University of Palermo, Via del Vespro 141, 90127 Palermo, Italy *Author for correspondence: Tel.: +39091 6552993 Fax: +39091 6552957 maurizio.averna@unipa.it 
(OMIM 603776, FH-type 3) [3]. FH-1 is a common disorder that affects approximately one in 500 individuals in the heterozygous form. FH-1 heterozygotes develop tendinous xanthomas, corneal arcus and coronary artery disease; the latter usually becomes evident in the fourth or fifth decade [4]. FH-1 homozygotes develop these features at an accelerated rate.

The rare FH-type 3, the most recently discovered form of $\mathrm{ADH}$, is caused by mutations of the PCSK9 gene [3].

Mutations in a gene called LDLRAP1 (also known as $A R H$ ), which serves as an adaptor for LDL-R endocytosis in the liver, result in a rare autosomal recessive form of FH known as autosomal recessive hypercholesterolemia (ARH - OMIM 608813) [5].

- Homozygous familial hypercholesterolemia $\mathrm{HoFH}$ is a rare inherited disease that has a prevalence worldwide of one in 1,000,000. In some geographic areas owing to a founder effect, the prevalence is higher: one in 30,000 in South Africa, one in 275,000 in Quebec, one in 100,000 in Lebanon and one in 171,167 in a Japan district [6-9]. Recent studies suggest a frequency as high as one in 200 for $\mathrm{HeFH}$ and this implies that it may be an underestimation of diagnosis in the general population, and the prevalence of $\mathrm{HoFH}$ may be higher than previously thought $[10,11]$.

HoFH patients may be true homozygotes if they carry two alleles with the same mutation of one of the three responsible genes or compound heterozygotes if they carry two alleles with two different mutations of the same gene; $\mathrm{HoFH}$ carriers of two heterozygous mutations of two different $\mathrm{FH}$ genes (digenic $\mathrm{HoFH}$ or double heterozygous $\mathrm{HoFH}$ ) have also been described [12].

Sometimes $\mathrm{HeFH}$ patients exhibit a clinically and biochemically severe phenotype, which overlaps with one of $\mathrm{HoFH}$; we do not have yet the mechanistic explanation but still unknown causative genes or modifier genes could be responsible.

In the majority of cases, $\mathrm{HoFH}$ is caused by mutations of the LDLR gene; only a few rare cases of HoFH compound heterozygotes of PCSK9/LDLR [9] or homozygotes for defective $A P O B$ mutations have been described [13]. HoFH mutations of LDLR and PCSK9 genes can also be classified into two categories on the basis of the genotype-phenotype effects:
- Null mutations if the LDL-R residual activity is $<2 \%$;

- Defective mutations if the LDL-R residual activity ranges between 2 and $25 \%$.

The clinical phenotype of ARH is considered milder than that of receptor-negative $\mathrm{HoFH}$ and resembles that observed in receptor-defective HoFH [5].

The clinical and biochemical phenotype of $\mathrm{HoFH}$ is characterized by the presence of:

- Very high levels of total and LDL-C (500-1000 mg/dl);

- Tendon xanthomas;

- Very early (first to second decade of life) clinical manifestations of coronary heart disease;

- Supravalvular aortic stenosis.

HoFH clinical severity, as well as atherosclerotic burden, are due to chronic exposure (since birth) to very high cholesterol levels. Receptor null $\mathrm{HoFH}$ patients are exposed to higher plasma cholesterol levels and have a higher risk of developing clinical events earlier than receptor defective HoFH patients.

\section{Available therapies: LDL apheresis}

LDL apheresis is an effective way to temporarily remove circulating ApoB-containing lipoproteins, including LDL, lipoprotein(a) (Lp[a]), and VLDL from the circulating blood. Although the majority of patients with hyperlipidemia can be sufficiently treated with dietary modification and drug therapy, LDL apheresis is indicated as first line treatment and approved by the FDA for patients with $\mathrm{HoFH}$, and to date, has been considered to be the only therapeutic option available to improve significantly the life expectancy of $\mathrm{HoFH}$ patients [14,15].

Over the years, the indication for apheresis has been also extended and approved for $\mathrm{HeFH}$ patients. Eligible $\mathrm{HeFH}$ patients include those who are not able to achieve an LDL-C recommended goal after 6 months of maximum tolerated hypolipidemic therapy, and also have LDL-C $>300 \mathrm{mg} / \mathrm{dl}$ or LDL-C $>200 \mathrm{mg} / \mathrm{dl}$ plus two cardiovascular risk factors or LDL-C $>160 \mathrm{mg} / \mathrm{dl}$ and a very high-risk cardiovascular profile $[16,17]$.

Different apheresis techniques have been developed over the years, including dextran sulphate cellulose adsorption, heparin-induced extracorporeal LDL-C precipitation, immunoadsorption and double filtration plasmapheresis 
of lipoproteins $[18,19,103]$. The two approved methods by the FDA are the LiposorberVR system and the heparin-induced extracorporeal LDL-C precipitation system [14].

Regardless of the apheresis technique used, the achievable acute reduction in LDL-C with a single procedure generally range from 50 to $75 \%$ from baseline per session. However, after apheresis, LDL-C levels rebound to pretreatment levels within 2-4 weeks [20].

The rapid drop in LDL-C with each LDL apheresis treatment does result in a reduction in mean LDL-C over time and this can be estimated by determining the pre- and post-apheresis LDL-C values, which can be used to estimate the time-averaged area under the rebound curve [20].

The goal of LDL-apheresis treatment is to reduce the time-averaged LDL-C by $\geq 60 \%$ of baseline or to reduce the total cholesterol by $>50 \%$ of baseline [20]. It has been demonstrated that in order to achieve this goal each LDL apheresis procedure should produce a reduction of total cholesterol $\geq 65 \%$ or LDL of $\geq 70 \%$ [20-23].

In addition, the time-averaged LDL-C is also influenced by the frequency of the procedures [20]. The usual frequency of procedures is every 2 weeks, but the treatment schedule may be shortened in case of inadequate response (weekly procedures) or delayed in patients with good response [20,23].

Recently, Graesdal and colleagues evaluated the effects of weekly LDL apheresis and maximum tolerated LLTs in seven HoFH patients on quality of life and cardiovascular status [24]. Although these patients were able to reach an LDL-C interval mean of $163 \mathrm{mg} / \mathrm{dl}$ and the frequency of procedures was well tolerated, progression of cardiovascular disease still occurred [24]. These results highlight the need for additional new therapies of which there are many in development.

\section{- Available therapies: statins \& cholesterol lowering agents}

In HoFH patients, statin therapy is less effective than in $\mathrm{HeFH}$ owing to the relative or absolute lack of LDL-Rs. However, statins remain a cornerstone of current pharmacological therapy for $\mathrm{HoFH}$ patients. In HoFH patients, the LDL-C reduction achieved with statins is mainly due to reduction in hepatic cholesterol synthesis. This effect explains why it is possible to obtain a marginal, but still important, cholesterol reduction.
High doses of simvastatin, atorvastatin and rosuvastatin have been demonstrated to reduce LDL-C by up to $48 \%$ with a great variability depending on the receptor status (negative or defective) [25-27]. The use of statins in monotherapy or in combination with other cholesterol lowering agents, despite a relatively modest LDL-C reduction, has been demonstrated to be effective in terms of years of life gained and the number of major atherosclerotic cardiovascular events spared [28].

HoFH patients belong to a very high-risk category for several reasons:

- Chronic exposure to very high cholesterol levels from birth;

- Early onset of the manifestations of coronary heart disease;

- Very poor outcome if untreated. Current treatment guidelines recommend an LDL-C goal of $<70 \mathrm{mg} / \mathrm{dl}$.

Although this target may not be achievable with every patient, it is reasonable to make a concerted effort to reach the lowest level of LDL-C possible (at least a $50 \%$ reduction) with any combination of both pharmacological (at the highest tolerated doses) and apheretic techniques [16]. Bile acid sequestrants, ezetimibe and niacin can be used in combination with statins in HoFH patients, and it is advisable to start the treatments as early as possible with the aim of keeping a life time cholesterol mean as low as possible. However, HoFH patients treated according to the current standard of care have never reached the recommended LDL-C goals and they continue to experience major atherosclerotic coronary events.

\section{- New drugs}

Recently, a number of new therapies have been developed that rely on novel strategies to lower LDL-C in patients with HoFH and/or HeFH. These therapies include mipomersen, inhibitors of PCSK9 and cholesteryl ester transfer protein (CETP), as well as lomitapide, a MTP inhibitor, the focus of this review.

Figure I schematically reviews the mechanisms of actions of hypocholesterolemic drugs.

\section{Mipomersen}

Mipomersen (mipomersen sodium, Kynamro ${ }^{\mathrm{TM}}$, Genzyme, MA, USA), developed under the name ISIS 301012, is a second-generation antisense oligonucleotide complementary and specific to 


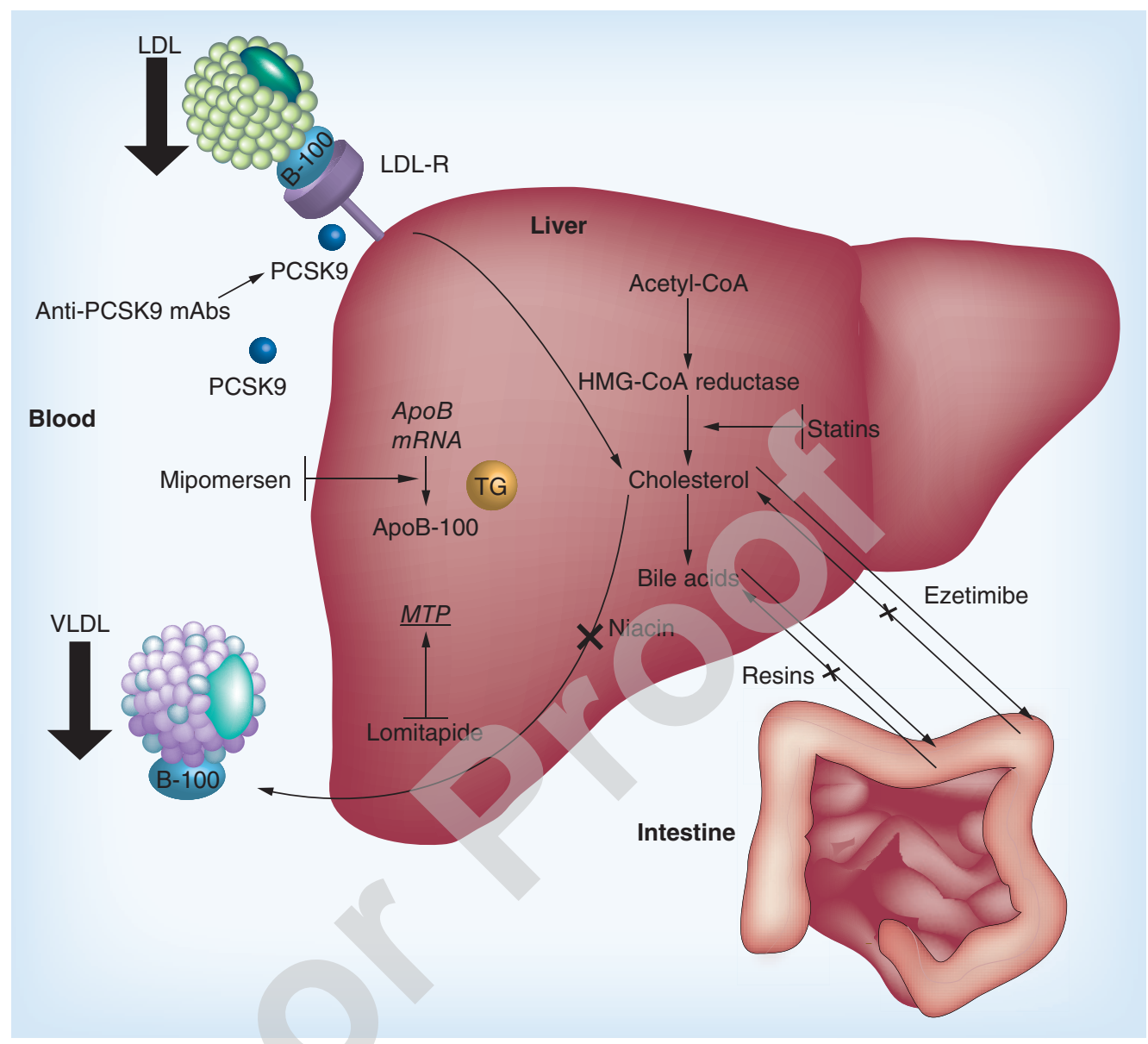

Figure 1. Mechanisms of action of hypocholesterolemic drugs.

HMG: Hydroxy-methylglutaryl; LDL-R: LDL receptor; mAb: Monoclonal antibody; MTP: Microsomal transfer protein; TG: Triglyceride.

human ApoB-100 mRNA. After its administration by subcutaneous injection in a formulation with $0.9 \%$ sodium chloride, mipomersen acts mainly in the liver, where it binds to ApoB mRNA causing its cleavage by the action of $\mathrm{RNase} \mathrm{H}$ and prevents the synthesis of ApoB-100 protein.

The efficacy of mipomersen was first tested in 36 volunteers with mild dyslipidemia [29]. In this study a dose-dependent reduction of serum ApoB and LDL-C was shown; at 200-mg dose, $A p o B$ and LDL-C were respectively reduced by 50 and $35 \%$. Levels of LDL-C below the baseline values were maintained for up to 3 months after the last administered dose [29].

Subsequently, Phase II and III trials evaluated mipomersen in patients with both $\mathrm{HeFH}$ and in $\mathrm{HoFH}$. In $\mathrm{HeFH}$, taking conventional LLT, mipomersen further reduced plasma $A p o B$ and LDL-C concentrations by a third [30]. Moreover, in statin-intolerant patients, including those with $\mathrm{HeFH}$ monotherapy with mipomersen demonstrated a significant reduction of LDL-C and ApoB (47 and 46\%, respectively) [31].
In the Phase III setting, Raal et al. assessed the efficacy and safety of $200 \mathrm{mg} /$ week of mipomersen in patients with $\mathrm{HoFH}$ in a randomized, double-blind, placebo-controlled trial [32]. In the mipomersen group who was already receiving the maximum tolerated dose of a lipidlowering drug except apheresis, a mean reduction of LDL-C of $25 \%$ was observed. However, there was a considerable broad range of variability in LDL-C changes $(2-82 \%)$ observed that appeared to be independent of baseline LDL-C levels, age, race or gender.

An interesting finding from the studies with mipomersen is the observation of $L p(a)$ reduction seen in mipomersen-treated patients, while TGs and HDL cholesterol levels were not significantly affected by mipomersen treatment [30].

Phase II and III studies have shown a satisfactory safety profile of mipomersen. The most common adverse event (AE) was injection site reactions in a substantial number of patients characterized by erythematous lesions occurring within $24 \mathrm{~h}$ of drug injection. Although 
injection site reactions are not considered to be serious AEs they may affect patient compliance. Flu-like symptoms have been observed in up to $70 \%$ of treated patients with mipomersen [31].

Finally, one of the concerns is that in some individuals mipomersen increases hepatic fat and it is not known what the long-term consequences are from this drug-induced hepatic steatosis.

Mipomersen has been approved by the FDA for the treatment of $\mathrm{HoFH}$ in the USA, while it is not approved by EMA in the EU.

\section{PCSK9 inhibitors}

PCSK9 is a serine protease, which is expressed in hepatocytes, kidney mesenchymal cells, intestine as well as in embryonic brain telencephalon neurons [33]. PCSK9 has a central role in regulation of cholesterol homeostasis by enhancing the endosomal and lysosomal degradation of hepatic LDL-R [34].

Although several approaches have been pursued to inhibit PCSK9 for treatment of hypercholesterolemia, the more successful thus far appear to be those observed in the Phase I and II clinical trials of human monoclonal antibodies (mAbs) targeting PCSK9.

The fully humanized mAb SAR236553/ REGN727 (Sanofi/Regeneron) has been shown to be effective in reducing LDL-C in healthy individuals $(33-46 \%)[35,36]$, as well as in patients with $\mathrm{HeFH}$ and hypercholesterolemic individuals without $\mathrm{FH}$ in conjunction with statins (40-72 and 38-66\%, respectively) [37,38].

Similar reductions in LDL-C have also been observed with another fully humanized antibody AMG145 Amgen (up to $64 \%$ relative to placebo in healthy individuals) [39]. In patients with $\mathrm{FH}$, the administration of $140 \mathrm{mg}$ of AMG145 antibody every 2 weeks reduced LDL-C by $51 \%$ [40], and in patients treated with statins with or without ezetimibe, AMG145 decreased LDL-C levels in a dose-dependent fashion by up to $66 \%[41,42]$.

Thus, both the SAR236553/REGN727 and AMG145 mAbs infused every 2 weeks appear to display similar efficacies in terms of LDL-C reduction both as monotherapy or in conjuction with statins. Importantly, in these studies, treatment resulted in the achievement of optimal target levels of LDL-C $(<70 \mathrm{mg} / \mathrm{dl})$ in a majority of the HeFH patients enrolled in these studies [35,40].

The TESLA proof-of-concept study was designed to evaluate the safety, tolerability and efficacy of AMG 145 in subjects with HoFH in which it is unknown if PCSK9 inhibition may work [104].
Eight $\mathrm{HoFH}$ patients with null or defective mutations of the LDLR were recruited (see 'Homozygous familial hypercholesterolemia') and treated with AMG145 (420 mg every 4 weeks for $\geq 12$ weeks, followed by every 2 weeks for an additional 12 weeks) [43].

After 12 weeks treatment, a $16.5 \%$ of LDL-C mean reduction from baseline was observed in the treated patients. However, the patients who experienced significant reduction of LDL-C (up to $43.6 \%$ from baseline) were carriers of defective mutations in the $L D L R$ gene, while no significant change from baseline were observed in $\mathrm{HoFH}$ patients carriers of null mutations (receptor-negative patients) [43].

These results suggest, for the first time, that PCSK9 $\mathrm{mAbs}$ may be useful in lowering LDL-C in HoFH carriers of defective mutations in the LDLR gene.

Although the results of these studies are promising, the ongoing Phase III PCSK9 mAbs clinical trials will provide more information on efficacy and safety including the risk of immune complex-mediated disorder related to long-term administration of mAbs.

\section{CETP inhibitors}

Inhibition of CETP is a target to increase HDL cholesterol and potentially correct abnormalities in HDL functionality in patients with $\mathrm{HoFH}$ [44].

The administration of anacetrapib to patients already on statin therapy has been demonstrated to further reduce LDL-C by approximately $40 \%$ and increase HDL cholesterol by approximately $140 \%$ [45]. The ongoing clinical trial on homozygous hypercholesterolemia will show the possible application in severe forms of hypercholesterolemia [105].

\section{Overview of MTP inhibition: lomitapide \\ - Pathophisiology of MTP}

MTP is an intracellular lipid-transfer protein essential for the assembly and secretion of VLDL particles in hepatocytes and chylomicron particles in enterocytes by initiating the incorporation of lipids into $A p o B$, and by acting as a chaperone to assist in the proper folding of the ApoB protein [46]. The role of MTP in lipid transport and metabolism was revealed by studies demonstrating that a genetic defect in the $M T P$ gene causes abetalipoproteinemia, an autosomal recessive disorder characterized by lack of production of ApoB-containing lipoproteins (chylomicrons and VLDL). 
Abetalipoproteinemia is a rare autosomal recessive disease; affected patients exhibit almost undetectable levels of ApoB and very low plasma cholesterol levels. Owing to the impairment of lipoprotein assembly, patients have fat malabsorption, steatorrhea and fat accumulation in enterocytes and hepatocytes $[46,47]$. The steatorrhea is usually minimized as subjects learn to avoid fat-rich diets at an early age. Subjects also suffer from multiple vitamin deficiencies (E, A, K and $\mathrm{D}$ ) because these fat soluble vitamins require the intestinal TG-rich ApoB-containing lipoproteins for normal absorption and transport. If untreated, patients with this disease will develop various pathologies including spinal-cerebellar degeneration, peripheral neuropathies and retinitis pigmentosa. These conditions can be prevented early in life by using fat soluble vitamin supplementation [47].

As newly synthesized ApoB is translocated into the lumen of the endoplasmic reticulum, it cotranslationally associates with a lipid to form a small nascent, lipid poor, lipoprotein particle. MTP helps initiate the transfer of lipids to ApoB. Both roles are believed to be important in promoting lipoprotein production [48]. The lipid transfer activity associated with MTP is crucial for lipoprotein assembly and secretion in hepatocytes and enterocytes [48,49]. Since MTP is highly expressed in the intestine, its inhibition may result in gastrointestinal (GI) disturbances, including diarrhea and steatorrhea, among others [50]. Studies demonstrating that defects in MTP cause abetalipoproteinemia suggested that inhibition of MTP could be a novel new mechanism to lower plasma lipid levels [48]. One would predict that MTP inhibitors would be capable of

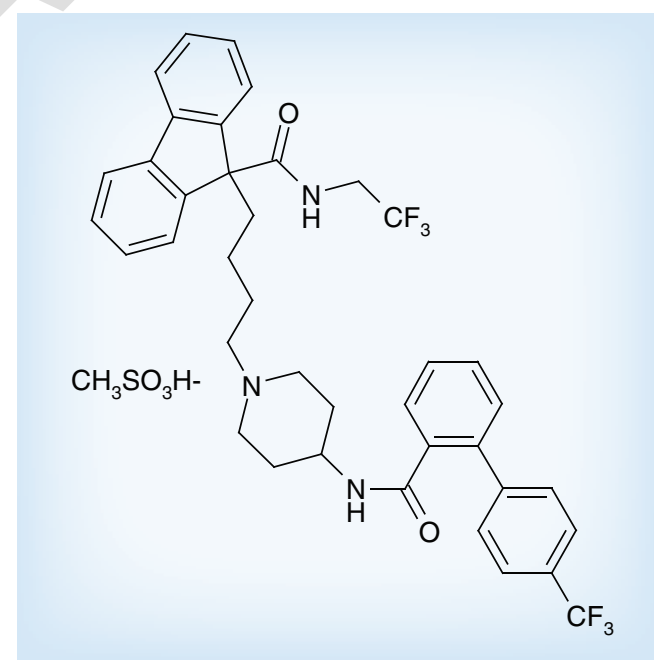

Figure 2. Chemical structure of lomitapide. inhibiting the production of both VLDL and chylomicrons. In support of this hypothesis, it has been recently reported that inhibitors of MTP activity, inhibit lipoprotein secretion in HepG2 cells [50,51]. Pharmacological inhibition of MTP may represent a novel therapeutic strategy to treat more severe forms of hyperlipidemia [52].

\section{- Pharmacological characteristics of lomitapide}

In an attempt to target MTP in order to reduce ApoB containing lipoproteins in patients with hypercholesterolemia, a series of small molecule benzimidazole-based analogs have been identified through different methodologies [53]. Intense efforts, using both conventional and combinatorial synthetic methods, led to the development of a compound named BMS-201038, which was investigated as candidate drug for the treatment of severe hypercholesterolemias.

This compound, now refered to as lomitapide (BMS-201038, AEGR-733, Juxtapid ${ }^{\circledR}$ in the USA and Lojuxta ${ }^{\circledR}$ in the EU), is a synthetic small molecule benzimidazole-based analog (Figure 2).

\section{Pharmacodynamics}

In cell culture studies, the $\mathrm{IC}_{50}$ for inhibition of ApoB secretion by lomitapide was much lower than that for ApoAI secretion $(0.8 \mathrm{nM}$ vs $6.5 \mu \mathrm{M})$, indicating that the compound is a highly selective inhibitor of ApoB secretion [54]. It was also noted that the secretion of ApoB from cultured HepG2 cells was significantly reduced compared with that in untreated cells [54].

\section{Pharmacokinetics}

Lomitapide shows a low absolute bioavailability of approximately $7 \%$, which has been attributed to a first-pass effect and the pharmacokinetics of lomitapide approach linearity at steady state. Pharmacokinetics appear approximately doseproportional between 10 and $50 \mathrm{mg}$ following multiple dose oral administration [106]. The drug is approximately $99.8 \%$ bound to plasma proteins [106]. The mean terminal half-life of lomitapide is approximately $39.7 \mathrm{~h}$ [106]

Lomitapide undergoes extensive metabolism in the liver via pathways that include oxidation, oxidative $N$-dealkylation, piperidine ring opening and glucuronide conjugation. CYP450 3A4 is involved in the metabolism of lomitapide to its major inactive metabolites M1 and M3. After administration of a single oral $60-\mathrm{mg}$ dose of lomitapide, the time to reach $\mathrm{C}_{\max }$ was approximately $6 \mathrm{~h}$ in healthy volunteers [106]. 


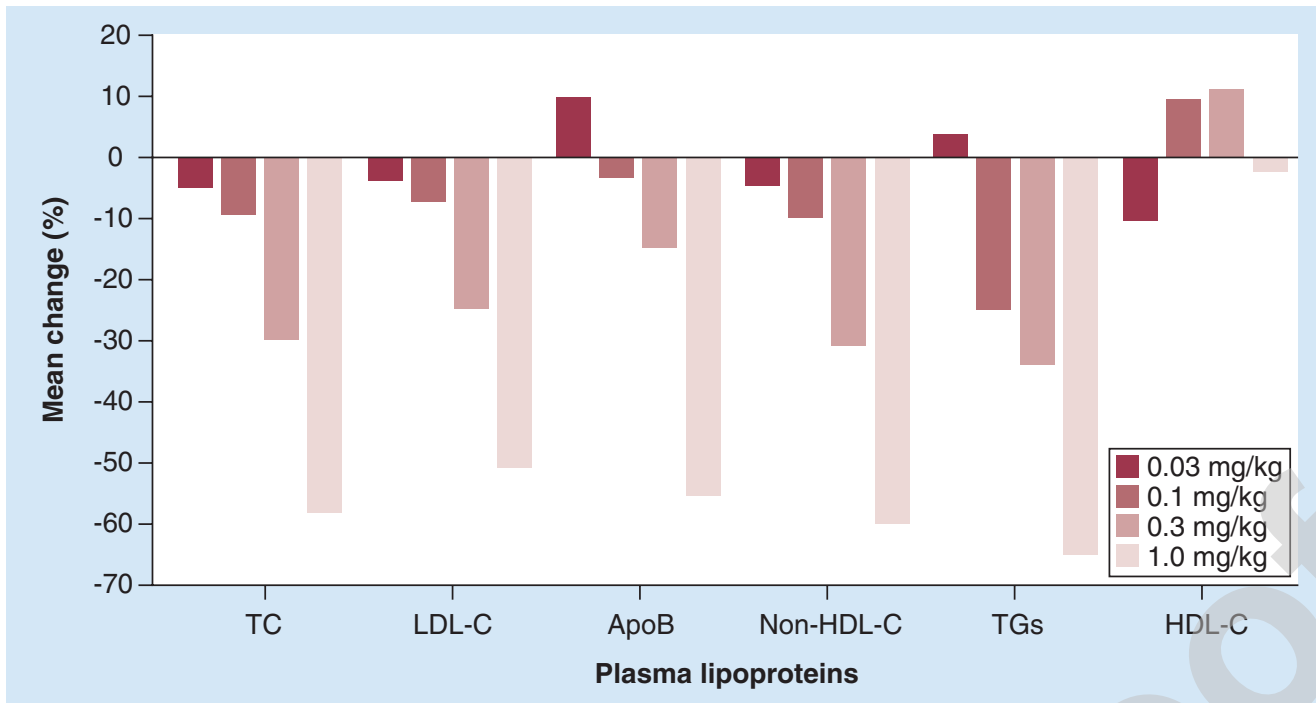

Figure 3. Mean percentage change in lipoproteins in the six homozygous familial hypercholesterolemia patients enrolled in the Phase II study.

HDL-C: HDL cholesterol; LDL-C: LDL cholesterol; TC: Total cholesterol; TG: Triglyceride.

Lomitapide is excreted both in the urine and feces (52.9-59.5 and 33.4-35.1\%, respectively) [106]. M1 is the major urinary metabolite and lomitapide is undetectable in urine samples. Lomitapide is the major component in the feces.

The pharmacokinetics of lomitapide have been evaluated in patients with end-stage renal disease receiving dialysis, but not in patients with end-stage renal disease not yet receiving dialysis or in patients with mild, moderate or severe renal impairment [104].

Pharmacokinetic interactions have been described between lomitapide and other CYP3A4 inhibitors. Coadministration of lomitapide with strong and moderate CYP3A4 inhibitors is contraindicated. When lomitapide is coadministered with simvastatin, the dose of the statin has to be reduced; coadministration with warfarin warrants more strict international normalized ratio monitoring [106].

\section{Clinical efficacy}

\section{- Phase II studies}

Lomitapide in moderate hypercholesterolemias In patients at moderate or high risk for coronary artery disease, it may be problematic to achieve recommended LDL-C levels due to either intolerance or inadequate response to available LLT.

On this basis, lomitapide was evaluated in a Phase II double-blind, placebo-controlled trial in 85 hypercholesterolemic patients at moderate or high risk [55]. In this trial, lomitapide $5-\mathrm{mg}$ daily for the first 4 weeks, 7.5 -mg daily for the second 4 weeks and 10-mg daily for the last 4 weeks) was dose-titrated and administered alone or in combination with ezetimibe $10-\mathrm{mg}$ daily.

In patients who were treated with lomitapide alone, a significant reduction of LDL-C and ApoB (30 and 24\%, respectively) was observed at a dose of $10 \mathrm{mg} /$ day. Combination therapy produced similar, but larger, dose-dependent decreases of LDL-C decrease ( $46 \%$ at $10 \mathrm{mg} /$ day of both lomitapide and ezetimibe). However, $16 \%$ of patients administered lomitapide, either alone or in combination with ezetimibe and none of the patients administered ezetimibe alone, discontinued the study drugs owing to mild elevations in transaminase elevation [55].

\section{Lomitapide in $\mathrm{HoFH}$ patients}

Preclinical studies conducted in Watanabe-heritable hyperlipidemic rabbits, which are a model for human $\mathrm{HoFH}[56,57]$ and $L D L R^{-/-}$mice models [58], supported the idea that MTP inhibition might be a viable approach to the treatment of this severe metabolic disorder.

The efficacy of oral lomitapide in the treatment of adults (aged $\geq 18$ years) with HoFH has been evaluated in a 16 week Phase II dose-escalation study of lomitapide monotherapy [59]. This Phase II study was nonrandomized, single-arm and open-labelled in design, and included six patients aged $18-40$ years with a clinical diagnosis of $\mathrm{HoFH}$, which was confirmed by genetic analysis. Congestive heart failure, major surgery within the previous 3 months, a history of 


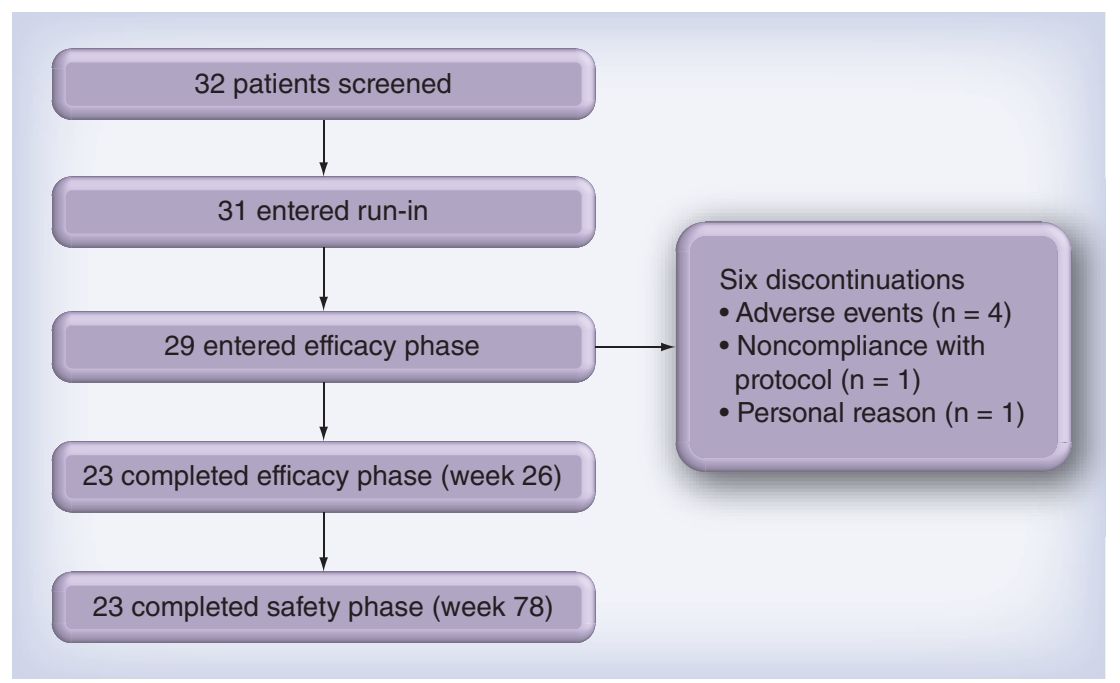

Figure 4. Phase III trial: the number of patients screened and the number of patients who completed the study.

hepatic disease, elevated aminotransferase levels, cancer within the 5 years before the start of the study, and a history of alcohol or drug abuse were among the criteria for exclusion from the trial. At least 4 weeks before the start of the study, LLTs, including apheresis, were discontinued and were not permitted at any time during the study; however, other drug treatment was allowed. At the initial screening evaluation, the six enrolled patients started a very low-fat diet $(<10 \%$ energy from fat) as well as standard multivitamins supplementation; and dietary counseling was provided during the study. Lomitapide was administered once-daily at escalating doses: $0.03,0.1$ and $0.3 \mathrm{mg} / \mathrm{kg} / \mathrm{day}$, then $1.0 \mathrm{mg} / \mathrm{kg} / \mathrm{day}$, each for 4 weeks. At baseline, the mean LDL-C level was $15.9 \mathrm{mmol} / \mathrm{l}(614 \mathrm{mg} / \mathrm{dl})$. In Figure 3, the mean changes in lipid and lipoprotein levels during the study are shown.

LDL-C and ApoB levels were reduced in a dose-dependent manner reaching the highest significant reduction at the highest titrated dose of $1.0 \mathrm{mg} / \mathrm{kg} /$ day (50.9 and $55.6 \%$, respectively).
The highest dose reductions in total cholesterol and TGs mean plasma levels were of 58.4 and $65.2 \%$, respectively (Figure 3). No significant changes in the plasma levels of HDL cholesterol were observed.

During the study, the most frequent drugrelated AEs were primarily GI, particularly transient increased stool frequency mostly linked to consumption of a high-fat meal. During the study, a broad range of caloric intake from fat was observed $(10-30 \%)$ and this may have influenced the GI tolerability of the drug.

Transaminase elevations were observed in four out of the six patients. In addition, hepatic fat content measured by MRI increased substantially in four patients in response to treatment with lomitapide (in two patients the hepatic fat content increase was $>30 \%$ ).

However, transaminases and hepatic fat levels returned to baseline levels 4 weeks after the therapy was stopped, with the exception of one patient in whom levels did not return to the normal range until 14 weeks after cessation of therapy.

\section{Phase III studies}

The efficacy and safety of lomitapide as adjunctive treatment to LLTs with or without LDL apheresis in patients with $\mathrm{HoFH}$ has been evaluated in a multicenter Phase III open-label trial [60]. In total, 29 adult ( $>18$ years) patients clinically and genetically diagnosed with $\mathrm{HoFH}$ ( 28 subjects were either true homozygotes or compound heterozygotes for mutations in the $L D L R$ gene and one was homozygous for an ARH [LDLRAP1] gene mutation) were enrolled in the trial and 23 completed the study (completers) (Figure 4). Week 26 represented the primary efficacy end point and week 78 the primary safety end point. The mean baseline LDL-C was $336 \pm 114 \mathrm{mg} / \mathrm{dl}$. During the first 26 weeks, the lomitapide dose was escalated from $5 \mathrm{mg}$ up

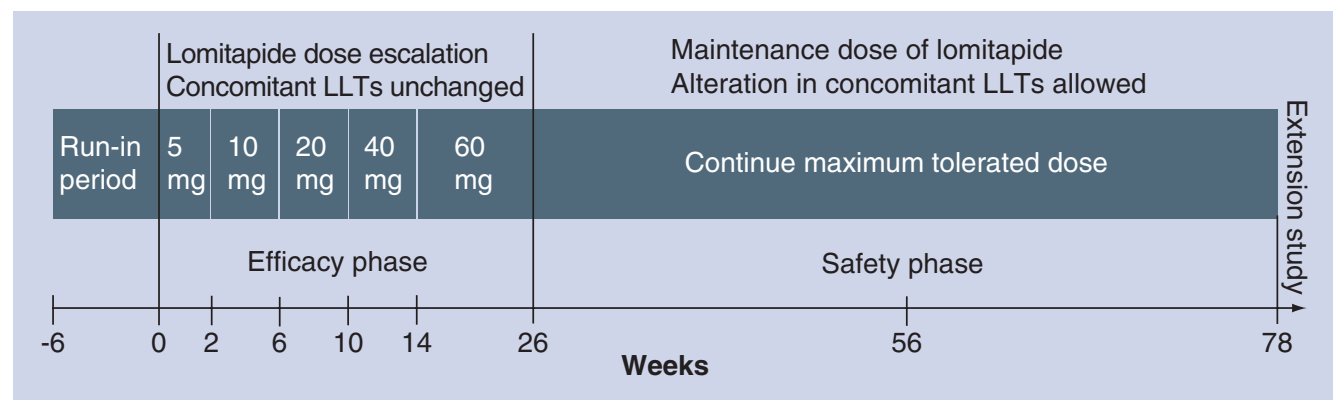

Figure 5. Phase III study design.

LLT: Lipid-lowering therapy. 
to a maximum dose of $60 \mathrm{mg} / \mathrm{day}$, in addition to current standard pharmacological therapy, which included lipoprotein apheresis (where available) without modifying the concomitant therapy (Figure 5).

The median dose of lomitapide of $40 \mathrm{mg}$ in the intent-to-treat population and $60 \mathrm{mg}$ in the completers was obtained. Six patients withdrew during the first 26 weeks, four due to AEs, one due to noncompliance and one for personal reasons (Figure 4). Additional end points were ApoB (49\%) and TGs (45\%) in the completers.

At the end of the safety period (week 78), the reduction of LDL-C, ApoB, TGs and nonHDL cholesterol was still significant $(38,43,31$ and $40 \%$, respectively) (Figure 6). This efficacy attenuation was likely due to the changes of the concomitant standard treatment allowed in the safety period. Interestingly, the Phase III trial demonstrated that at one or more point on treatment with lomitapide, $55 \%$ of $\mathrm{HoFH}$ patients $(\mathrm{n}=16)$ reached the LDL-C goal of $<100 \mathrm{mg} / \mathrm{dl}$ and $31 \%(n=9)$ reached the LDL-C goal of $<70 \mathrm{mg} / \mathrm{dl}$. Moreover, six patients interrupted or reduced the frequency of lipoprotein apheresis treatments because of an important reduction of LDL-C. There was also a $15 \%$ reduction of $\mathrm{Lp}$ (a) at week 26, which reverted back to baseline by week 78 . The reasons for this are yet to be understood but may relate to the changes in background therapy permitted from week 26 to week 78. Table I summarizes the Phase III trial results.

A post hoc intention-to-treat-analysis of the influence of the apheresis on the efficacy of lomitapide did not show significant differences between $\mathrm{HoFH}$ patients receiving $(\mathrm{n}=18)$ or not receiving $(\mathrm{n}=11)$ lipoprotein apheresis [61].

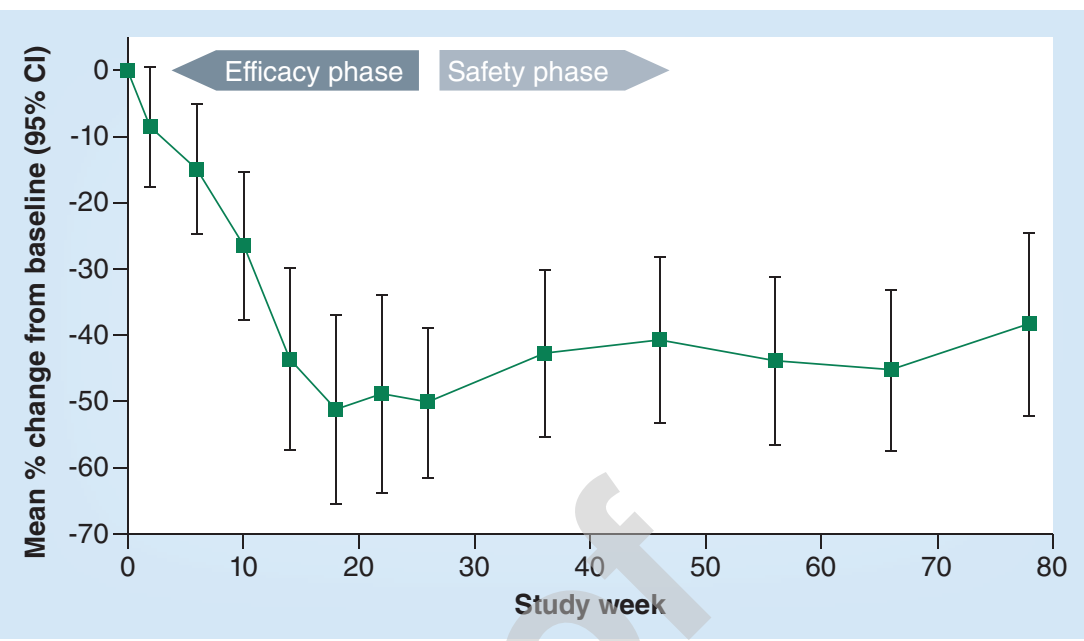

Figure 6. Change in LDL cholesterol during week 78 in patients who completed the study (those who completed the study: $\mathbf{n}=23$ ).

\section{Safety \& tolerability}

Lomitapide proof-of-concept and Phase II studies have shown that a high dose of the drug was associated with severe and frequent hepatic and GI side effects, both due to the drug mechanism of action [55,59]. The dose-escalation strategy adopted in the Phase III trial combined with the administration of a low-fat diet and the supplementation of lipid-soluble vitamins and essential fatty acids, was aimed to achieve the optimal balance between efficacy and safety. The Phase III trial results at the safety end point (week 78) indicate that overall lomitapide is safe and well tolerated [60]. The AEs were mainly GI including nausea, heartburn, flatulence, diarrhea and fecal urgency; three out of 29 enrolled patients discontinued because of the GI AEs during the first 6 months, but all of the patients who entered the safety phase completed the study $(\mathrm{n}=23)$. The majority of the patients in the efficacy

\section{Table 1. Comparison of characteristics of lomitapide with mipomersen.}

\begin{tabular}{|c|c|c|c|c|}
\hline Drug & Mechanism & $\begin{array}{l}\text { Main lipid } \\
\text { effect }\end{array}$ & Adverse effects & Comments \\
\hline Lomitapide & $\begin{array}{l}\text { Reduced secretion of } \\
\text { ApoB-containing } \\
\text { lipoproteins from the } \\
\text { liver by MTP inhibition }\end{array}$ & $\begin{array}{l}\text { Reduction of: } \\
\text { LDL-C }(50 \%) \\
\text { ApoB }(49 \%) \\
\text { TG }(45 \%)\end{array}$ & $\begin{array}{l}\text { Hepatic fat } \\
\text { accumulation } \\
\text { Gastrointestinal side } \\
\text { effects } \\
\text { Elevation of liver } \\
\text { function tests }\end{array}$ & $\begin{array}{l}\text { US FDA and EMA approval for HoFH } \\
\text { Has been used in primary hypercholesterolemia } \\
\text { patients on top of conventional LLT }\end{array}$ \\
\hline
\end{tabular}




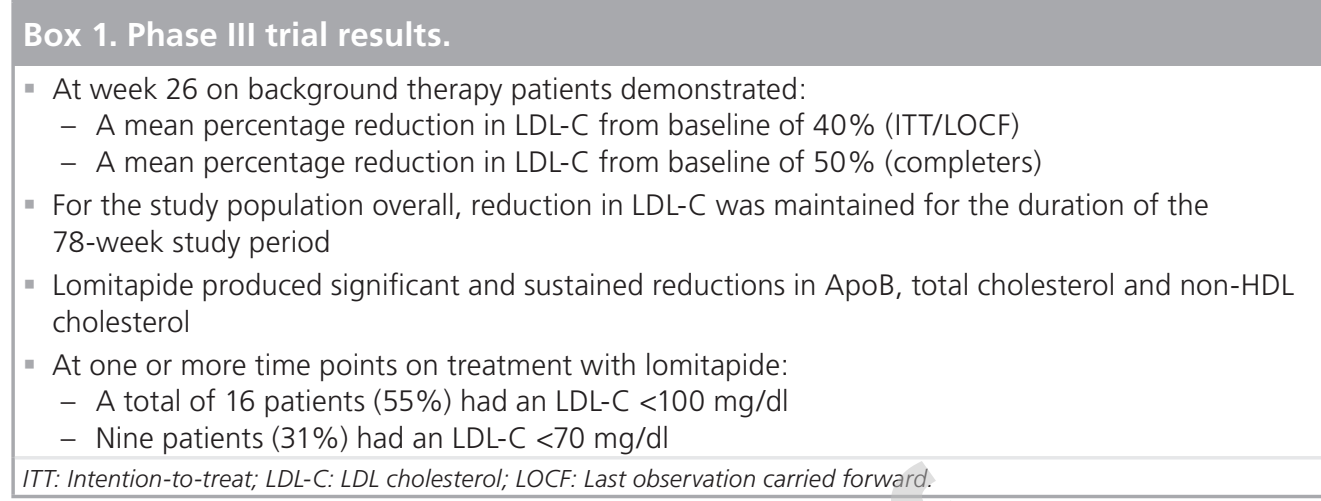

phase experienced GI AEs of mild or moderate intensity. Interestingly, over time, a decrease in the frequency and intensity of GI AEs was observed, maybe due to an intestinal adaptation to the drug or to a better self-management of the dietary fat intake. The BMI did not change during the trial and there was no evidence of net malabsorption of fat soluble nutrients. The transaminases (alanine transaminase and/or aspartate transaminase) levels in the majority of patients remained $<2 \times$ upper limit of normal (ULN); only three patients had ALT and/or AST levels $>5 \times$ ULN $-\leq 10 \times$ ULN and one patient $>10 \times$ ULN $-\leq 20 \times$ ULN. No patient showed changes in bilirubin and alkaline phosphatase levels. The elevations of hepatic enzymes were managed according to the trial protocol with the dose reduction or temporary drug discontinuation, and no patient permanently discontinued the trial. Alcohol consumption above the protocolspecified limits was a possible cause in three of the four cases. Lomitapide, by inhibiting MTP, reduces the hepatic TGs export leading to fatty liver. In the Phase III trial, hepatic fat accumulation was monitored by nuclear magnetic resonance spectroscopy. During the trial period, the hepatic fat content increased from $1 \%$ at baseline to $8.6 \%$ at end of the efficacy end point (week 26), remaining stable at the end of safety end point (week 78). During the entire trial, only one patient showed a hepatic fat content $>20 \%$ to $\leq 25 \%$ and two patients $>25 \%$.

Overall, lomitapide was well tolerated and the benefits in terms of reduction of LDL-C in $\mathrm{HoFH}$, a very high-risk patient population outweigh the potential risks associated with the hepatic and intestinal mechanism of action of the drug (Box 2).

\section{Lomitapide in clinical practice}

Lomitapide is indicated only in adult patients with $\mathrm{HoFH}$ in addition to a low-fat diet, other lipid-lowering medications and with or without LDL apheresis treatment. Treatment should be initiated and monitored by a physician experienced in the treatment of lipid disorders.

The recommended starting dose is $5 \mathrm{mg}$ oncedaily and the dose should be escalated gradually to minimize the incidence and severity of GI side effects (after 2 weeks to $10 \mathrm{mg}$ and then, at a minimum of 4-week intervals, to $20,40 \mathrm{mg}$, and to the maximum recommended dose of $60 \mathrm{mg}$ ).

\section{Box 2. Safety summary of the Phase III trial.}

\section{Gastrointenstinal adverse events}

\section{- Most common reason for discontinuation}

- Four out of total six discontinuations: 14\% overall; all subjects noncompliant with diet

- Usually of mild or moderate intensity; Gl adverse reactions of severe intensity were reported by six (21\%) out of 29 patients

- The reported incidence and number of patients who experienced Gl events was lower during the safety phase

\section{Hepatic AEs}

- Four out of 29 patients experienced transient and reversible ALT elevations $\geq 5 x$ ULN that were managed by dose reduction or temporary interruption

- ALT/AST elevations were clinically manageable using the dose-reduction/dose interruption algorithm specified in the study protocol

- No significant elevations in bilirubin or alkaline phosphatase were observed

- No Hy's law cases

- Hepatic fat increased modestly during the dose-escalation phase and then stabilized

No subjects discontinued lomitapide treatment based on liver function test elevations or hepatic related adverse events.

AE: Adverse events; ALT: Alanine aminotransferase; AST: Aspartate aminotransferase; GI: Gastrointestinal; ULN: Upper limit of normal. 
Since the administration of lomitapide with food may influence GI tolerability the drug should be taken on an empty stomach at least $2 \mathrm{~h}$ after the evening meal.

In order to minimize GI adverse reactions associated with the use lomitapide patients should follow a low fat diet ( $<20 \%$ of energy from fat) and dietary counseling should be provided.

Based on observations of decreased essential fatty acid and vitamin E levels in clinical trials, patients should take daily dietary supplements that provide 400-IU vitamin E, and at least 200-mg linoleic acid, 110-mg eicosapentaenoic acid, 210-mg $\alpha$-linolenic acid and 80-mg docosahexaenoic acid per day, throughout treatment with lomitapide.

Lomitapide is contraindicated in patients with moderate or severe hepatic impairment and patients with mild hepatic impairment (Child-Pugh A) should not exceed 40-mg daily.

Regular liver monitoring should be performed at baseline and on an annual basis using both imaging and biomarker evaluations; if aminotransferase elevations are accompanied by clinical symptoms of liver injury, increases in bilirubin $\geq 2 \times U L N$, or active liver disease, treatment with lomitapide must be discontinued and the patient referred to a hepatologist for further investigation.

Drinking alcohol may increase the risk of liver side effects: in the medication guide issued by the EMA, the use of alcohol during lomitapide treatment is not recommended while the FDA limit the consumption of alcoholic drink to one each day.

Patients should avoid consumption of grapefruit juice and if a concomitant treatment with a weak CYP3A4 inhibitor is indicated the dose of lomitapide should be reduced and the dose of lomitapide should be up-titrated according to LDL-C response and safety/tolerability.

Concomitant use of moderate or strong CYP3A4 inhibitors with lomitapide is contraindicated and if treatment with these class of drugs is unavoidable, lomitapide should be stopped during the course of treatment.

Coadministration of a CYP3A4 inducer is expected to reduce the effect of lomitapide and it is recommended to increase the frequency of LDL-C assessment during such concomitant use, and should consider increasing the dose of lomitapide to ensure maintenance of the

\section{Executive summary}

\section{Mechanism of action}

- Lomitapide binds and inhibits microsomal triglyceride transfer proteins, thereby inhibiting the synthesis and secretion of triglyceride-rich chylomicrons in the intestine and VLDL in the liver.

\section{Pharmacokinetics \& metabolism}

- Lomitapide is primarily metabolized by the liver via pathways that include oxidation and glucuronide conjugation. Lomitapide is mainly metabolized by CYP3A4 to its inactive metabolites, M1 and M3. Approximately $52.9-59.5 \%$ is eliminated by the urine and $33.4-35.1 \%$ is eliminated by the feces. Lomitapide half-life is approximately $39.7 \mathrm{~h}$.

\section{Clinical efficacy}

- As adjunctive treatment of homozygous familial hypercholesterolemia already treated with standard therapy, including lipoprotein apheresis reduced LDL cholesterol by $50 \%$, ApoB by $49 \%$ and triglycerides by $45 \%$ after 26 weeks in a Phase III trial.

- After a further 52-week treatment period (safety phase), the reduction of LDL-C, ApoB, triglycerides and non-HDL cholesterol was still significant $(38,43,31$ and 39\%, respectively). This efficacy attenuation may be due to the changes of the concomitant standard treatment.

- Six of the 13 patients receiving apheresis during the safety phase were able to discontinue or reduce the frequency of the lipoprotein apheresis treatments.

\section{Safety \& tolerability}

- Overall, lomitapide was well tolerated with side effects related to the action of the drug.

\section{Gastrointestinal adverse events}

- Nausea, heartburn, flatulence, diarrhea and fecal urgency mainly of mild or moderate intensity and dose dependent.

- Decreased over time of frequency and intensity due to possible intestinal adaptation to the drug and/or a better self-management of the dietary fat intake.

\section{Liver adverse events}

- Increase of transaminases (alanine transaminase/aspartate transaminase).

- Increase of hepatic fat content.

\section{Dosage \& administration}

- Suggested starting dose is dose $5 \mathrm{mg}$ in combination with current standard pharmacological therapy, including lipoprotein apheresis (where available). Increase titration up to maximum dose of $60 \mathrm{mg} /$ day to individualize therapy. 
desired level of efficacy if the CYP3A4 inducer is intended for chronic use.

\section{Regulatory affairs}

On December 2012, the FDA approved lomitapide as an orphan drug for use in adult patients with $\mathrm{HoFH}$ in combination with other LLTs. Lomitapide is available in the USA only through a restricted program called the JUXTAPID Risk Evaluation and Mitigation Strategy Program.

The EMA has approved lomitapide $\left(\right.$ Lojuxta $\left.^{\circledR}\right)$ for the same indication.

\section{Future perspective}

Treatment with lipoprotein apheresis and hypolipidemic drugs have improved the management of patients with $\mathrm{HoFH}$, but the clinical outcomes are still severe. The available evidence suggests that lomitapide may represent an effective and safe adjunctive therapy for the management of $\mathrm{HoFH}$.

It is reasonable to expect that, in the future, an increasing number of $\mathrm{HoFH}$ patients will be treated with lomitapide and that more evidence on the long-term safety will be available. Evidence is needed on the possible benefits of an early start of the lomitapide treatment in $\mathrm{HoFH}$ pediatric patients. The ultimate long-term challenge for lomitapide will be to show a reduction of cardiovascular events and mortality in $\mathrm{HoFH}$.

\section{Financial \& competing interests disclosure}

$A B$ Cefalù, MD Panno and MR Averna have served as clinical investigators in several hypolipidemic drug trials for Aegerion, Merck, Pfizer, Sanofi and Astra Zeneca. MR Averna is a member of Aegerion Europe and Italy Advisory Boards. The manufacturer of the drug that is the primary focus of this manuscript was offered an opportunity to comment on this article; comments provided were only related to medical accuracy and completeness. Any changes resulting from comments received were made by the authors on the basis of scientific and/or editorial merit. The authors have no other relevant affiliations or financial involvement with any organization or entity with a financial interest in or financial conflict with the subject matter or materials discussed in the manuscript apart from those disclosed.

No writing assistance was utilized in the production of this manuscript.

\section{References}

Papers of special note have been highlighted as: "n of considerable interest

1 Lomitapide. Am. J. Cardiovasc. Drugs 11(5), 347-352 (2011).

2 Innerarity TL, Weisgraber KH, Arnold KS et al. Familial defective apolipoprotein B-100: low density lipoproteins with abnormal receptor binding. Proc. Natl Acad. Sci. 84(19), 6919-6923 (1987).

3 Abifadel M, Varret M, Rabes JP et al. Mutations in PCSK9 cause autosomal dominant hypercholesterolemia. Nat. Genet. 34(2), 154-156 (2003).

4 Goldstein JL, Hobbs HH, Brown MS et al. Familial Hypercholesterolemia. In: The Methabolic and Molecular Bases of Inherited Disease (8th Edition). Scriver CR (Ed.). Mc Graw-Hill, NY, USA, 2863-2913 (2001).

5 Pisciotta L, Priore Oliva C, Pes GM et al. Autosomal recessive hypercholesterolemia (ARH) and homozygous familial hypercholesterolemia (FH): a phenotypic comparison. Atherosclerosis 188(2), 398-405 (2006).

6 Seftel HC, Baker SG, Sandler MP et al. A host of hypercholesterolaemic homozygotes in South Africa. Br. Med. J. 281(6241), 633-636 (1980).

7 Moorjani S, Roy M, Gagne C et al. Homozygous familial hypercholesterolemia among French Canadians in Quebec Province. Arteriosclerosis 9(2), 211-216 (1989).

Fahed AC, Safa RM, Haddad FF et al. Homozygous familial hypercholesterolemia in Lebanon: a genotype/phenotype correlation. Mol. Genet. Metab. 102(2), 181-188 (2011).

9 Mabuchi H, Nohara A, Noguchi T et al. Molecular genetic epidemiology of homozygous familial hypercholesterolemia in the Hokuriku district of Japan. Atherosclerosis 214(2), 404-407 (2011).

10 Benn M, Watts GF, Tybjaerg-Hansen A et al. Familial hypercholesterolemia in the Danish general population: prevalence, coronary artery disease, and cholesterol-lowering medication. J. Clin. Endocrinol. Metab. 97(11), 3956-3964 (2012).

11 Nordestgaard BG, Chapman MJ, Humphries SE et al.; for the European Atherosclerosis Society Consensus Panel. Familial hypercholesterolaemia is underdiagnosed and undertreated in the general population: guidance for clinicians to prevent coronary heart disease: Consensus Statement of the European Atherosclerosis Society. Eur. Heart J. 34(45), 3478a-3490a (2013).

12 Ejarque I, Civer M, Francisco Ascaso J et al. Identification and characterization of the first Spanish familial ligand-defective apolipoprotein B homozygote Med. Clin. (Barc.) 116(4), 138-141 (2001).

13 Pisciotta L, Priore Oliva C, Cefalu AB et al. Additive effect of mutations in $L D L R$ and PCSK9 genes on the phenotype of familial hypercholesterolemia. Atherosclerosis 186(2), 433-440 (2006).

14 Sanchez AP, Cunard R, Ward DM. The selective therapeutic apheresis procedures. J. Clin. Apher. 28(1), 20-29 (2013).

15 Thompson GR, Miller JP, Breslow JL. Improved survival of patients with homozygous familial hypercholesterolaemia treated with plasma exchange. Br. Med. J. (Clin. Res. Ed.) 291(6510), 1671-1673 (1985).

16 Robinson JG. Management of familial hypercholesterolemia: a review of the recommendations from the National Lipid Association Expert Panel on Familial Hypercholesterolemia. J. Manag. Care Pharm. 19(2), 139-149 (2013).

17 Ito MK, McGowan MP, Moriarty PM. Management of familial hypercholesterolemias in adult patients: recommendations from the National Lipid Association Expert Panel on Familial Hypercholesterolemia. J. Clin. Lipidol. 5(Suppl. 3), S38-S45 (2011).

18 Winters JL. Lipid apheresis, indications, and principles. J. Clin. Apher. 26(5), 269-275 (2011). 
19 Bambauer R, Bambauer C, Lehmann B et al. LDL-apheresis: technical and clinical aspects. ScientificWorldJournal 2012, 314283 (2012).

20 Thompson GR; HEART-UK LDL Apheresis Working Group. Recommendations for the use of LDL apheresis. Atherosclerosis 198(2), 247-255 (2008).

21 Thompson GR. LDL apheresis. Atherosclerosis 167(1), 1-13 (2003).

22 Hodgins LC, Gordon BR, Parker TS et al. LDL apheresis: an effective and safe treatment for refractory hypercholesterolemia. Cardiovasc. Drug Rev. 20(4), 271-280 (2002).

23 Thompson GR, Catapano A, Saheb S et al. Severe hypercholesterolaemia: therapeutic goals and eligibility criteria for LDL apheresis in Europe. Curr. Opin. Lipidol. 21(6), 492-498 (2010).

24 Græsdal A, Bogsrud MP, Holven KB et al. Apheresis in homozygous familial hypercholesterolemia: the results of a follow-up of all Norwegian patients with homozygous familial hypercholesterolemia. J. Clin. Lipidol 6(4), 331-339 (2012).

- Results on the weekly apheresis schedule and cardiovascular assessment in homozygous familial hypercholesterolemia (HoFH) patients.

25 Marais AD, Blom DJ, Firth JC. Statins in homozygous familial hypercholesterolemia. Curr. Atheroscler. Rep. 4(1), 19-25 (2002).

26 Raal FJ, Pilcher GJ, Illingworth DR et al. Expanded-dose simvastatin is effective in homozygous familial hypercholesterolaemia. Atherosclerosis 135(2), 249-256 (1997).

27 Marais AD, Raal FJ, Stein EA et al. A dose-titration and comparative study of rosuvastatin and atorvastatin in patients with homozygous familial hypercholesterolaemia. Atherosclerosis 197(1), 400-406 (2008).

28 Raal FJ, Pilcher GJ, Panz VR et al. Reduction in mortality in subjects with homozygous familial hypercholesterolemia associated with advances in lipid lowering therapy. Circulation 124(20), 2202-2207 (2011).

29 Kastelein JJ, Wedel MK, Baker BF et al. Potent reduction of apolipoprotein B and low-density lipoprotein cholesterol by short-term administration of an antisense inhibitor of apolipoprotein B. Circulation 114(16), 1729-1735 (2006).

30 Akdim F, Visser ME, Tribble DL et al. Effect of mipomersen, an apolipoprotein B synthesis inhibitor, on low-density lipoprotein cholesterol in patients with familial hypercholesterolemia. Am. J. Cardiol. 105(10), 1413-1419 (2010).
31 Visser ME, Wagener G, Baker BF et al. Mipomersen, an apolipoprotein B synthesis inhibitor, lowers low-density lipoprotein cholesterol in high-risk statin-intolerant patients: a randomized, double-blind, placebo-controlled trial. Eur. Heart J. 33(9), 1142-1149 (2012).

32 Raal FJ, Santos RD, Blom DJ et al. Mipomersen, an apolipoprotein B synthesis inhibitor, for lowering of LDL cholesterol concentrations in patients with homozygous familial hypercholesterolaemia: a randomised, double-blind, placebo-controlled trial. Lancet 375(9719), 998-1006 (2010).

33 Seidah NG, Benjannet S, Wickham L et al. The secretory proprotein convertase neural apoptosis-regulated convertase 1 (NARC-1): liver regeneration and neuronal differentiation. Proc. Natl Acad. Sci. USA 100 (3), 928-933 (2003).

34 Lambert G, Sjouke B, Choque B, Kastelein JJ, Hovingh GK. The PCSK9 decade. J. Lipid Res. 53(12), 2515-2524 (2012).

35 Stein EA, Mellis S, Yancopoulos GD et al. Effect of a monoclonal antibody to PCSK9 on LDL cholesterol. N. Engl J. Med. 366(12), 1108-1118 (2012).

36 Stein EA, Gipe D, Bergeron J et al. Effect of a monoclonal antibody to PCSK9, REGN727/ SAR236553, to reduce low-density lipoprotein cholesterol in patients with heterozygous familial hypercholesterolaemia on stable statin dose with or without ezetimibe therapy: a Phase 2 randomised controlled trial. Lancet 380 (9836), 29-36 (2012).

37 McKenney JM, Koren MJ, Kereiakes DJ et al. Safety and efficacy of a monoclonal antibody to proprotein convertase subtilisin/kexin type 9 serine protease, SAR236553/REGN727, in patients with primary hypercholesterolemia receiving ongoing stable atorvastatin therapy. J. Am. Coll. Cardiol. 59(25), 2344-2353 (2012).

38 Roth EM, McKenney JM, Hanotin C et al. Atorvastatin with or without an antibody to PCSK9 in primary hypercholesterolemia. N. Engl. J. Med. 367(20), 1891-1900 (2012).

39 Dias CS, Shaywitz AJ, Wasserman SM et al. Effects of AMG 145 on low density lipoprotein cholesterol levels: results from 2 randomized, double-blind, placebo-controlled, ascending-dose Phase 1 studies in healthy volunteers and hypercholesterolemic subjects on statins. J. Am. Coll. Cardiol. 60(19), 1888-1898 (2012).

40 Koren MJ, Scott R, Kim JB et al. Efficacy, safety, and tolerability of a monoclonal antibody to proprotein convertase subtilisin/ kexin type 9 as monotherapy in patients with hypercholesterolaemia (MENDEL): a randomised, double-blind, placebo-controlled, Phase 2 study. Lancet 380 (9858), 1995-2006 (2012).

41 Raal F, Scott R, Somaratne R et al. Low-density lipoprotein cholesterol lowering effects of AMG145, a monoclonal antibody to proprotein convertase subtilisin/kexin type 9 serine protease in patients with heterozygous familial hypercholesterolemia: the Reduction of LDL-C with PCSK9 Inhibition in Heterozygous Familial Hypercholesterolemia Disorder (RUTHERFORD) randomized trial. Circulation 126(20), 2408-2417 (2012).

42 Giugliano RP, Desai NR, Kohli P et al. Efficacy, safety, and tolerability of a monoclonal antibody to proprotein convertase subtilisin/kexin type 9 in combination with a statin in patients with hypercholesterolaemia (LAPLACE-TIMI 57): a randomised, placebo-controlled, dose-ranging, Phase 2 study. Lancet 380 (9858), 2007-2017 (2012).

43 Stein EA, Honarpour N, Wasserman SM, Xu F, Scott R, Raal F. Trial evaluating AMG145, a PCSK9 antibody, in patients with homozygous FH: results of an initial dose scheduling study. Presented at: Abstract Book of the 81th European Atherosclerosis Society Congress. Lyon, France, 2-5 June, 2013 (Abstract: 1625).

44 Guerin M. Reverse cholesterol transport in familial hypercholesterolemia. Curr. Opin. Lipidol. 23(4), 377-385 (2012).

45 Cannon CP, Shah S, Dansky HM et al. Safety of anacetrapib in patients with or at high risk for coronary heart disease. N. Engl. J. Med. 363(25), 2406-2415 (2010).

46 Raabe M, Véniant MM, Sullivan MA et al. Analysis of the role of microsomal triglyceride transfer protein in the liver of tissue-specific knockout mice. J. Clin. Invest. 103(9), 1287-1298 (1999).

47 Kane JP, Havel RJ, Scriver CR, Beaudet AL. The Metabolic Basis of Inherited Disease (6th Edition). Scriver CR (Ed.), McGraw-Hill, NY, USA (1989).

48 Wetterau JR, Lin MC, Jamil H. Microsomal triglyceride transfer protein. Biochim. Biophys. Acta 1435(2), 136-150 (1997).

49 Hussain MM, Rava P, Walsh M et al. Multiple functions of microsomal triglyceride transfer protein. Nutr. Metab. (Lond.). 9, 14 (2012).

" - Complete review on microsomal triglyceride transfer protein function.

50 Haghpassand M, Wilder D, Moberly JB. Inhibition of apolipoprotein B and triglyceride secretion in human hepatoma cells (HepG2). J. Lipid Res. 37(7), 1468-1480 (1996). 
51 Golberg CA. Emerging low-density lipoprotein therapies: microsomal triglyceride transfer protein inhibitors. J. Clin. Lipidol. 7(Suppl. 3), S16-S20 (2013).

52 Gordon DA, Jamil H. Progress towards understanding the role of microsomal triglyceride transfer protein in apolipoprotein-B lipoprotein assembly. Biochim. Biophys. Acta 1486(1), 72-83 (2000).

53 Sulsky R, Robl JA, Biller SA et al. Carboxamido-1,3,2-dioxaphosphorinanes, potent inhibitors of MTP. Bioorg. Med. Chem. Lett. 14(20), 5067-5070 (2004).

54 Robl JA, Sulsky R, Sun CQ et al. A novel series of highly potent benzimidazole-based microsomal triglyceride transfer protein inhibitors. J. Med. Chem. 44(6), 851-856 (2001).

55 Samaha FF, McKenney J, Bloedon LT et al. Inhibition of microsomal triglyceride transfer protein alone or with ezetimibe in patients with moderate hypercholesterolemia. Nat. Clin. Pract. Cardiovasc. Med. 5(8), 497-505 (2008).

56 Wetterau JR, Gregg RE, Harrity TW et al. An MTP inhibitor that normalizes atherogenic lipoprotein levels in WHHL rabbits. Science 282 (5389), 751-754 (1998).

57 Shiomi M, Ito T. MTP inhibitor decreases plasma cholesterol levels in LDL receptordeficient WHHL rabbits by lowering the
VLDL secretion. Eur. J. Pharmacol. 431(1), 127-131 (2001).

58 Liao W, Hui TY, Young SG et al. Blocking microsomal triglyceride transfer protein interferes with ApoB secretion without causing retention or stress in the ER. J. Lipid Res. 44(5), 978-985 (2003).

59 Cuchel M, Bloedon LT, Szapary PO et al. Inhibition of microsomal triglyceride transfer protein in familial hypercholesterolemia. N. Engl. J. Med. 356(2), 148-156 (2007).

w11. Proof-of-concept study on the efficacy of lomitapide in $\mathrm{HoFH}$ patients.

60 Cuchel M, Meagher EA, du Toit Theron H et al. Efficacy and safety of a microsomal triglyceride transfer protein inhibitor in patients with homozygous familial hypercholesterolaemia: a single-arm, open-label, Phase 3 study. Lancet 381(9860), 40-46 (2013).

"II This Phase III study in HoFH patients confirms the efficacy of lomitapide in reducing $\mathrm{LDL}$ cholesterol levels with acceptable safety and tolerability.

61 Cuchel M, Meagher EA, du Toit Theron H et al. Apheresis treatment does not affect the lipid-lowering efficacy of lomitapide, a microsomal triglyceride transfer protein inhibitor, in patients with homozygous familial hypercholesterolemia. Circulation 126, Abstract 17396 (2012).

\section{- Websites}

101 FDA Approved Drug products. www.accessdata.fda.gov/scripts/cder/ drugsatfda/index.cfm? fuseaction $=$ Search . DrugDetails

102 European Medicines Agency. Lojuxta. www.ema.europa.eu/ema/index. jsp?curl=pages $/$ medicines $/$ human $/$ medicines/002578/human_med_001668. jsp\&mid=WC0b01ac058001d124

103 Moga C, Hastall C. Low density lipoprotein apheresis for the treatment of familial hypercholesterolemia. Health Technology Assessment. IP-18 Information Paper. Alberta Heritage Foundation for Medical Research (April 2004). www.ihe.ca/documents/ip18.pdf

104 Trial Evaluating PCSK9 Antibody in Subjects With LDL Receptor Abnormalities (TESLA). April 2012.

http://clinicaltrials.gov/show/NCT01588496

105 Efficacy and Tolerability of Anacetrapib Added to Ongoing Lipid-Lowering Therapy in Adult Participants With Homozygous Familial Hypercholesterolemia (HoFH) (MK0859-0042 AM2). April 2013. http://clinicaltrials.gov/show/NCT01841684

106 Aegerion Pharmaceuticals Inc. US prescribing information for Juxtapid ${ }^{\mathrm{TM}}$ (lomitapide) 2013. www.juxtapid.com/_pdf/Prescribing_ Information.pdf 